\title{
L'évidence du complot : un défi à l'argumentation. Douter de tout pour ne plus douter du tout
}

The Evidence of Conspiracy: a Challenge to Argumentation. Doubting everything, so as not to doubt at all

\section{Loïc Nicolas}

\section{(2) OpenEdition}

\section{Journals}

Electronic version

URL: http://journals.openedition.org/aad/1833

DOI: 10.4000/aad.1833

ISSN: 1565-8961

\section{Publisher}

Université de Tel-Aviv

\section{Electronic reference}

Loïc Nicolas, «L'évidence du complot : un défi à l'argumentation. Douter de tout pour ne plus douter du tout », Argumentation et Analyse du Discours [Online], 13 | 2014, Online since 14 October 2014 connection on 23 September 2019. URL : http://journals.openedition.org/aad/1833 ; DOI : 10.4000/ aad. 1833

This text was automatically generated on 23 September 2019

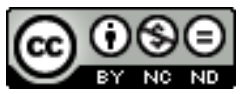

Argumentation \& analyse du discours est mis à disposition selon les termes de la licence Creative Commons Attribution - Pas d'Utilisation Commerciale - Pas de Modification 4.0 International. 


\title{
L'évidence du complot : un défi à l'argumentation. Douter de tout pour ne plus douter du tout
}

\author{
The Evidence of Conspiracy: a Challenge to Argumentation. Doubting everything, \\ so as not to doubt at all
}

\section{Loïc Nicolas}

Dans le prolongement des travaux menés en collaboration avec Emmanuelle Danblon (Danblon \& Nicolas 2010, 2012), cet article se propose d'analyser certains mécanismes langagiers et argumentatifs saillants à l'œuvre dans ce qu'on appelle, par habitude, les «théories du complot». Grands récits ou historiettes, ces «théories» traduisent (et bien souvent trahissent) une certaine tournure de l'esprit qui, tout en affichant son objectivité absolue, s'attache à mettre en cohérence des événements épars prélevés çà et là dans la réalité observable. Elles intègrent ces événements à l'intérieur d'une trame narrative déterministe (mais non assumée comme telle) capable de leur donner du sens et de l'épaisseur; capable, en somme, de les faire parler. Très concrètement: ces théories (1) recueillent et assemblent des événements élevés au statut de faits (Dominicy 2010), dans le but (2) d'apporter la preuve que ces faits sont nécessairement liés entre eux, (3) parce qu'ils résultent d'une cause unique, c'est-à-dire (4) d'un complot dont ils témoignent, et (5) au sein duquel les participants agissent conformément à une nature profonde qui les détermine. Ce travail de «mise en fiction », qui s'opère tous azimuts, livre alors un « tout » cohérent, massif et sécurisant, capable de relier ensemble le passé, le présent et le futur sans discontinuité. Mais la "fiction" produite, n'est fiction que de l'extérieur - pour tous ceux qui refusent d'adhérer à cette vision du monde. À l'intérieur de la théorie, il n'y a (par définition) ni conteurs, ni fabulistes, ni interprètes, seulement des hommes et des femmes de bonne volonté. Des spectateurs qui regardent simplement ce qui est avec lucidité et sans complaisance. Pour eux, tout est déjà-là. Il n'y a qu'à ouvrir les yeux et à prêter l'oreille.

2 En d'autres termes, les théories en question pointent et dévoilent les supposées raisons cachées qui justifient qu'on présente les faits collectés en un seul geste discursif, d'un 
seul trait. Aussi, les raisons invoquées s'élaborent-elles, d'une part, en évacuant complètement le rôle du hasard, du flou et du précaire qui habitent les affaires humaines, d'autre part, en sollicitant des explications où prédominent les intentions inavouables (et partant inavouées) de groupes d'individus motivés par une essence ou une propriété commune - juif, américain, franc-maçon, communiste, capitaliste, homosexuel, etc. Dès lors, censées converger vers les mêmes intérêts, les mêmes fins, les actions et les décisions des conspirateurs désignés répondent, forcément, à un plan concerté, un programme ou un protocole.

Ce faisant, l'explication par le complot se révèle simple et complexe à la fois. Simple, parce qu'elle permet d'ordonner au sein d'un récit unique et totalisant des éléments très divers, parfois contradictoires, de la réalité sociale pour les faire tenir ensemble. La logique conspirationniste se livre ainsi à de véritables tours de force. Elle réussit à faire feu de tout bois en passant outre les oppositions les plus fortes. Qu'on pense, par exemple, au titre d'un billet récemment publié sur le site de l'écrivain conspirationniste canadien Henry Makow, grand pourfendeur du Nouvel Ordre Mondial : « Adolf Hitler Agent of Zionism and Freemasonry ${ }^{1}$ ", ou encore à cette synthèse également improbable et déroutante, où politiciens de tous bords, capitalistes, socialistes, communistes, sionistes se retrouvent dans le même sac :

ORDO AB CHAO, devise si chère à l'Élite du NWO [New World Order]. Il y a une autre devise qui n'est pas souvent cité et fait référence aux systèmes politiques actuels et à venir aussi. Cette devise est : thèse + antithèse $=$ synthèse. Que veut donc dire cette devise ? Et bien tout simplement : Capitalisme + Socialisme = COMMUNISME. Le communisme et le socialisme sont une invention des hommes les plus riches de la planète. Hé oui les sionistes sont les instigateurs de ce plan macabre ${ }^{2}$.

C'est justement cette capacité unificatrice, cette plasticité même qui donne réponse à tout, que j'ai appelée ailleurs « rhétorique de la facilité » (Nicolas 2010). Toutefois, pour être «facile », l'explication par le complot n'en est pas moins profondément complexe. En effet, elle prétend dévoiler tout un maillage de connexions, de collusions, d'intérêts, de plans intermédiaires, qui sont structurés sur la base d'étroits rapports de causes à effets. La mise à jour de ce maillage, donc sa production, reposent sur l'accumulation d'indices, de signes, de traces plus ou moins visibles. Ceux-ci sont alors investis et interprétés à l'aune de l'explication totale ( = la vision du monde) sur laquelle repose tout l'édifice théorique. Ces indices, signes, traces (la disparition ou la présence d'un document, l'existence d'un lointain cousinage entre adversaires politiques, le silence des autorités, la contradiction ou l'univocité des sources... peu importe) constituent pour les dénonciateurs du complot autant de témoignages des manœuvres et des manigances qui se trament dans le monde souterrain. Au reste, comme le rappelle Gérald Bronner avec beaucoup de justesse à propos des sectes : dès lors qu'on part « en quête de signes, ils finissent toujours par arriver » (Bronner 2009 : 260). En effet, il n'est jamais difficile de trouver, dans la masse des informations disponibles, celles qui, justement, vont pouvoir confirmer la thèse initiale et renforcer la clôture du monde. Statistiquement, il n'est d'ailleurs pas «anormal» que des événements ou des coïncidences improbables aient lieu - même si cela déroute ou intrigue. À cet égard, Bronner montre combien le «biais de confirmation » constitue un mécanisme cognitif particulièrement efficace lorsqu'il s'agit de légitimer et de pérenniser ses propres croyances. L'esprit de celui qui adhère avec force à une thèse tend à se focaliser sur et, partant, à ne retenir que les faits (souvent spectaculaires) qui confirment ses attentes, «sans égard pour le poids de ceux qui les infirment ». Il n'est dès lors pas (ou plus) en 
mesure de comprendre que ses "trouvailles ne révèlent rien d'autre qu'une des productions possibles du hasard» (Bronner 2009 : 83).

5 Finalement, dans cette quête de sens et de confirmation, tout peut servir à lézarder le vernis (forcément) fragile de ces thèses que l'on dit « officielles ». Lesquelles n'auraient jamais d'autres buts que de tenir les profanes (à savoir tout un chacun) dans l'ignorance de ce qui a vraiment eu lieu ou qui se trame dans la réalité. Leur fonction serait de garantir la perpétuation du « système » (ce Léviathan des temps modernes) et de permettre à ceux qui complotent de continuer à comploter en paix. En tout état de cause, suivant un raisonnement de ce type, chaque nouvel indice (ou absence d'indice, car tout est caché, bien sûr), aussi ténu soit-il, constitue une preuve supplémentaire que le complot existe : une preuve irréfutable de son évidence. Or, comme le relèvent dès l'introduction les deux auteurs du Traité de l'Argumentation, " on ne délibère pas là où la solution est nécessaire et on n'argumente pas contre l'évidence » (Perelman \& Olbrechts-Tyteca 2000 [1958] : 1). Nul ne saurait lutter contre elle, à moins d'être fou, insensé, aveugle à l'égard des faits ou, comme nous pourrions le dire dans le cas présent, complice voire acteur de la conspiration.

6 Partant, celui qui dénonce - et, par-là, dévoile, met en lumière, montre la réalité et les faits bruts - se présente, plus qu'aucun autre pourrait-on croire, comme le garant des acquis et des exigences de la raison moderne. Son entreprise critique paraît légitime. En battant en brèche les vérités " autorisées » et les thèses officielles, le dénonciateur du complot cherche, semble-t-il, à ouvrir les yeux de ses contemporains. Il se donne pour mission de les déciller; de leur exposer l'évidence pour dissiper les ténèbres. Libre penseur incorruptible, expert-citoyen, son discours, dit-il, n'engage que lui, mais se fait, en même temps, le relais de tous ceux qui n'ont pas le loisir de parler publiquement - les sans-voix. Comprenons bien, la pensée conspirationniste en affichant un respect scrupuleux des contraintes de production du savoir scientifique (les faits, rien que les faits), profite de l'extraordinaire légitimité que nos sociétés démocratiques attachent à la position critique. Elle parvient alors, nouveau tour de force, à s'immuniser face à la critique même, par un recours systématique à l'effet d'évidence. C'est à ce titre qu'elle est si persuasive. Précisément, la pensée conspirationniste, à l'image des pensées extrêmes en général, a tendance à développer, pour reprendre l'idée de Bronner, « une forme de système immunitaire ». Ce système rend alors « la croyance invulnérable aux contradictions quelles qu'elles soient », et se traduit par un effet de "cliquet » qui, dès lors, « empêche le croyant de revenir en arrière » (Bronner 2009 : 310). On assiste à la formation d'une sorte de plafond de verre sur lequel la critique et les contradictions, dès lors, viennent buter.

7 Pour aller plus loin, cet article entend questionner la place accordée au doute dans la pensée conspirationniste et, plus encore, l'ambiguïté avec laquelle celui-ci est traité. C'est ici le point central : reconsidérer le type d'argumentation propre à cette pensée-là à la lumière du couple doute-évidence. En effet, dans un même geste, le doute qui envahit l'espace social (sur le mode du "tout n'est qu'apparences», ou du " on nous cache tout ») en vient à quitter totalement celui du discours à l'intérieur duquel, justement - évidence oblige -, on ne doute pas. Le monde dont ces discours rendent compte est à la fois ouvert (en prise aux forces occultes et terrifiantes de la conspiration) et rigoureusement clos. L'explication totale - celle qui fait tenir la vision du monde - se referme sur elle-même en renvoyant hors de la discussion, hors de la raison même, tous ceux qui ne voient pas (ou qui ne veulent pas voir) la réalité en face : 
c'est-à-dire le complot. Pour ses dénonciateurs, les preuves avancées sont forcément contraignantes et devraient s'imposer à tous sans possibilité de critique.

En conséquence, l'adhésion à ces preuves constitue un droit et non pas un fait. Le but n'est pas de rallier l'adversaire récalcitrant, mais bien de le combattre pour lui faire entendre raison. Nous y reviendrons par la suite. Or, nous le savons, il ne saurait y avoir de contrainte en rhétorique, ni pour adhérer à une thèse, ni pour retirer son adhésion. Aucune prémisse, aucune preuve, aucune procédure, aucun argument n'est suffisamment «fort » ou «bon» pour faire de l'adhésion un acte nécessaire. Comme l'écrivent Perelman et Olbrechts-Tyteca dans «Logique et rhétorique », leur article programmatique publié en 1950 , au sein

[d']une argumentation rhétorique tout peut toujours être remis en question; on peut toujours retirer son adhésion : ce que l'on accorde est un fait, non un droit. [...] On ne peut être obligé d'adhérer à une proposition ou obligé d'y renoncer à cause d'une proposition à laquelle on serait acculé. L'argumentation rhétorique n'est pas contraignante parce qu'elle ne se déroule pas à l'intérieur d'un système dont les prémisses et les règles de déduction sont univoques et fixées de manière invariable (Perelman \& Olbrechts-Tyteca, 1952 [1950] : 26).

Concrètement, le raisonnement conspirationniste crée un espace qui semble ouvert au doute et à l'argumentation, alors même qu'il rend l'expression de l'un comme de l'autre impossible. La finalité de ce type de discours n'est nullement d'assumer le doute, ni d'en faire une ressource dans la démarche argumentative. Au contraire, tout semble fait pour lui retirer sa dimension pratique, mais aussi pour dissiper la part incompressible d'incertitude et de jeu qui permet à la liberté de choix d'opérer. Douter de tout pour ne plus douter du tout : telle pourrait être la devise d'une vidéo mise en ligne le 11 septembre 2011 par le cinéaste Mathieu Kassovitz. Ce dernier s'y fait le relais des théories du complot propres aux attentats perpétrés dix ans plus tôt. Il reprend la plupart des topoï du discours conspirationniste - bien qu'il s'en défende avec force. L'enregistrement d'une quinzaine de minutes pourrait mériter une analyse suivie, je ne proposerai là qu'un extrait en guise de cas d'école :

Admettre que quelque chose ne va pas dans la thèse officielle, comprendre la façon dont évidemment elle a été fabriquée, [...] c'est un travail sur [soi] que beaucoup de gens ne sont pas capables de faire, que la majorité n'est pas capable de faire. [...] La négation de la réalité c'est la négation de la science. [...] Vous avez le droit de vous poser des questions. [...] Si vous avez des doutes, vous avez le droit de les émettre. [...] Nous sommes des millions à avoir des doutes, il y a des milliers de spécialistes qui se sont regroupés main dans la main pour comprendre ce qui s'est passé. Et ils ont trouvé, ils savent. Écoutons-les³.

10 Tout y est : l'aveuglement du plus grand nombre (tous ceux qui ne sont " pas capables » de voir ou d'accepter la réalité, partant, la fabrication des "thèses officielles »); la masse indistincte des lucides (des milliers, des millions peut-être ?) qui ont fait « un travail» personnel et se posent des questions; l'affirmation d'être dans son bon «droit» en doutant; l'impossibilité d'échapper à la vérité des faits (à moins de souhaiter rester dans l'erreur); l'appel à la science généreuse (celle des «spécialistes [...] regroupés main dans la main »); l'effet d'évidence... Pour le dire sans détour, il ne s'agit pas de se "poser des questions ", ni même d'argumenter, mais bien de confirmer une matière qui est déjà jugée, à savoir que tout est « fabriqué ».

11 Dans la suite de cette contribution, je développerai mon propos en trois temps. J'aborderai tout d'abord l'articulation entre le doute et le choix dans la pensée de Chaïm Perelman, puis je mènerai une investigation sur la place de la critique dans 
l'argumentation rhétorique, enfin je proposerai, en m'appuyant sur une série d'exemples, un bref panorama de la topique conspirationniste.

\section{L'articulation du doute et du choix chez Chaïm Perelman}

12 L'entreprise de persuasion n'a de sens qu'à partir du moment où le problème soulevé, la question à trancher, la chose à juger, la décision à prendre, demeurent dans une incertitude telle que (1) l'argumentation puisse se déployer sur le mode de l'invention, et (2) que le fait d'être persuadé puisse constituer un acte libre et responsable. En d'autres termes, la rhétorique repose sur un doute fondateur, une indistinction, un flou, dont on peut dire qu'ils font sa raison d'être. S'il n'y avait pas ce doute primordial, cette incertitude première quant au chemin à suivre, si tout était parfaitement évident, il n'y aurait aucune bonne raison de partir en quête d'arguments, ni de chercher à persuader quiconque. D'ailleurs, comme le souligne Aristote au premier Livre de sa Rhétorique (I, 1357 a 4-7) :

Nous ne délibérons que sur les questions qui sont manifestement susceptibles de recevoir deux solutions opposées; quant aux choses qui, dans le passé, l'avenir ou le présent ne sauraient être autrement, nul n'en délibère, s'il les juge telles; car cela ne lui servirait à rien. (Aristote $1960:$ 79)

13 Cet extrait bien connu d'Aristote peut être mis en regard avec un passage lui aussi fameux du livre III (chapitre cinq) de l'Éthique à Nicomaque concernant les matières sujettes ou non à délibération : « Est-ce qu'on délibère sur toutes choses, autrement dit est-ce que toute chose est objet de délibération? Nous devons sans doute appeler un objet de délibération non pas ce sur quoi délibérerait un imbécile, ou un fou, mais ce sur quoi peut délibérer un homme sain d'esprit.». Aristote y pointe notamment l'importance de l'incertitude: non pas une incertitude radicale sur base de laquelle nous ne pourrions rien dire de raisonnable, mais une incertitude qui donne prise à l'argumentation. Partant, les « objets » qui (1) dépendent de nous, (2) que nous pouvons potentiellement réaliser, et dont (3) l'issue est indéterminée, sont suffisamment incertains, sans l'être totalement, pour faire l'objet d'une investigation proprement rhétorique. Pour autant, si l'incertitude et le doute animent ou accompagnent la démarche rhétorique, se font jour une série de questions cruciales à savoir : que faire du doute inaugural ? Quel rôle lui donner ? comment le traiter en discours ? S'agit-il de l'occulter, de le dissoudre dans une certitude, ou encore de le réduire par l'argumentation? Peut-on concilier doute et décision? Et, en fin de compte, comment faire la part des choses, c'est-à-dire dissocier le doute sur lequel repose le cheminement argumentatif, de celui qu'on trouve à l'œuvre dans la pensée conspirationniste ?

14 Pour aborder ces questions, je m'appuierai sur le texte d'une conférence de Perelman qui préfigure (et en un sens informe) la réflexion menée par ce dernier sur la rhétorique. Ce texte, publié en 1949, précède d'une dizaine d'années la parution du Traité, et d'un an celle de l'article programmatique (1950) que j'ai précédemment cité. À l'époque de cette conférence sur le « libre examen », Perelman a déjà entendu parler de rhétorique, certes - depuis peu de temps toutefois -, mais il n'en a pas encore fait la clé de voûte de sa réflexion. Pourtant, il a une intuition née sur les ruines de la Deuxième 
Guerre mondiale ; une intuition relative au doute, mais aussi à l'intrication d'éléments épars qu'il veut réunir : le libre examen, la parole persuasive, l'incertitude, l'orientation dans un monde flou, la décision. En tout état de cause, Perelman se demande comment faire pour conférer au doute une dimension pratique, pour lui donner force et vigueur afin d'éviter qu'il ne sclérose la pensée, la parole et l'action :

À la maxime fasciste : «croire, obéir, combattre », nous en opposons une autre qui serait: «Douter, se décider et convaincre », maxime qui oppose le primat de la pensée à celui de la force. Remarquons combien cette règle est difficile, car elle fait tout d'abord appel à l'initiative de l'individu, et à son sentiment de responsabilité, parce qu'il ne suffit pas de douter, de nier, de détruire ; dans la vie il faut toujours savoir agir, c'est-à-dire prendre une décision, et il est bien souvent difficile de prendre une décision [...]. Il est aussi plus souvent facile de combattre un adversaire que de le convaincre, car il n'est pas du tout certain que l'on réussira à le convaincre. (Perelman 2009 [1949] : 146)

Trois points doivent, selon moi, nous interpeller dans cet extrait remarquable. D'abord, Perelman refuse de réduire le doute à une posture - sociale ou discursive. Laquelle pourrait alors l'apparenter à une nouvelle croyance qui serait nihiliste et destructrice. Ce que Perelman refuse catégoriquement par ailleurs. Ensuite, l'auteur lie le doute et l'action : à une action personnelle (délibérer avec soi-même pour se décider), d'une part; à une action vers autrui (convaincre, nous aurions préféré persuader, mais peu importe), d'autre part. Enfin, Perelman n'invite nullement à renoncer au doute. Il propose, à l'inverse, de l'assumer : d'en faire une occasion, un kairos.

Pourquoi convaincre, justement, ce n'est pas combattre? Pour Perelman, prendre "l'initiative ", donc le risque (car rien n'est gagné d'avance), de convaincre, c'est: (1) reconnaitre la précarité même des arguments avancés à l'intention d'autrui, leur caractère critiquable et, dans un même mouvement (c'est le corrélat), (2) accepter de s'exposer soi-même aux arguments qu'autrui pourrait également formuler. Chercher à persuader (ou à convaincre), c'est aussi prendre le risque d'être persuadé (ou convaincu). L'un ne va pas sans l'autre, puisque le doute résiste : il est là. Lequel doute n'empêche pas de choisir, c'est-à-dire de se donner des raisons capables de motiver et de justifier la décision prise. Mais il oblige à adosser ses raisons à un sens du risque, donc de la responsabilité. Perelman développe cette idée dans son article consacré au "problème du bon choix »:

C'est parce qu'il n'y a pas de règles susceptibles de fournir une solution définitive au problème du bon choix que chaque choix constitue un risque, une option engageant la responsabilité de l'homme qui a choisi, que l'appréciation morale a un sens et que l'on peut parler de liberté humaine. (Perelman 1952 [1948]:160)

La liberté dont il est question ici est intimement liée à la démarche rhétorique. Celle-ci ne saurait d'aucune façon se déployer dans un monde clos, ni dans un système où il s'agirait de «transformer [1]es consciences humaines en automates ajustés aux besoins d'une idéologie" (Perelman 1952 [1948]: 160). C'est à ce titre que la pensée conspirationniste entretient un rapport profondément ambigu (même s'il est revendiqué comme clair) tant à la liberté qu'à la responsabilité. Et ceci dans la mesure où elle refuse d'assumer les risques inhérents aux choix qu'elle pose et aux interprétations qu'elle formule quant aux événements du monde. Des choix et des interprétations qui, finalement, n'en sont pas vraiment puisqu'il n'existe, pour sûr, aucune possibilité raisonnable d'agir ni de lire le monde autrement. L'acte délibératif est, par définition, sans objet, puisque l'adhésion est inconditionnelle (Bronner 2009: 118-132) - tout du moins dans les cas extrêmes, sachant qu'il existe des niveaux 
d'adhésion variés. Aussi les théories du complot parviennent-elles à soustraire leurs adeptes à la responsabilité qui incombe à tous ceux qui décident de s'engager et de donner du sens. Elles les libèrent de leurs choix et des risques dont ceux-ci sont porteurs. L'effet produit est alors celui d'une grande sécurité cognitive. Partant, la liberté, affichée comme un étendard par ceux qui relayent ce type de discours, n'est tout au plus qu'une façade pour masquer un refus de se soumettre à la critique. Laquelle, forcément malvenue, confronte à l'inacceptable, à savoir : «l'infirmité de la certitude ». Elle laisse croire que le monde humain est « ambigu [et] ne porte pas en lui le dernier mot » (Gusdorf 1960 : 94-95); que le complot n'est pas inscrit dans la nature des choses ni dans celle des hommes.

À présent, je vais m'efforcer de montrer dans quelle mesure la critique constitue un moteur de l'argumentation, ou plus exactement un outil à l'intérieur du voyage rhétorique.

\section{Critique et rhétorique}

19 En pénétrant dans l'espace d'opposition des preuves et des arguments, les adversaires (réels ou potentiels) contractent, l'un envers l'autre, une obligation qui les met en devoir de se respecter comme tels, c'est-à-dire comme adversaires. Suivant une perspective non normative de l'argumentation, la relation qu'ils instaurent ne repose pas sur la négociation (d'une distance quelconque), ni sur la coopération (il ne s'agit pas de rechercher le consensus, l'accord ou l'union des contraires), mais sur une forme de coordination minimale qui rend possible leur rencontre discursive et les opérations critiques qui en découlent. Cela veut dire que s'ils choisissent de s'affronter par les mots (y compris de façon virulente), c'est qu'ils voient, dans l'affrontement même, un lieu (légitime et rationnel) pour assumer de concert l'ambiguïté du monde. Le désaccord suppose, lui aussi, un accord des esprits quant à l'existence d'une ambiguïté sur laquelle le désaccord porte.

Très concrètement, cette coordination minimale est relative, d'une part à ce qui fait l'objet du différend, sans pour autant que les adversaires attribuent à cet objet une définition identique, et d'autre part à la façon, non pas de le régler, mais bien d'en explorer les implications, la matière, les enjeux. À cet égard, la réversibilité des rôles et des places entre celui qui formule sa critique et celui qui y répond est constitutive de cette «invention défensive » dont parle Sebastian McEvoy dans l'ouvrage éponyme (1995). Invention au nom de laquelle chacun des protagonistes se charge successivement de se défendre et d'accuser, c'est-à-dire de rechercher (et donc de prendre en charge) les preuves les mieux à même de supporter une défense toujours à venir. La réversibilité en question est inhérente au processus argumentatif au sein duquel une preuve qui parait acceptable et persuasive pour l'un, peut être entièrement refusée par un autre, appelant alors une argumentation en sens opposé qui inverse la charge de la preuve. La recherche des preuves et, partant, leur administration en vue de les faire admettre comme des raisons, et même de «bonnes raisons » d'adhérer est la première charge des protagonistes de l'interaction, par laquelle ils manifestent, justement, leur raison pratique.

21 En ce sens, il y a toujours, dans la démarche argumentative-persuasive qui fonde l'opposition des adversaires, un périlleux engagement de la parole: un risque intimement lié à cette recherche qui n'est jamais assurée d'aboutir. Or c'est justement 
dans cet engagement que s'enracine le paradigme rhétorique. Un paradigme qui donne prise et consistance à un monde vivant, sachant qu'un "monde sans risque serait un monde mort ; la parfaite assurance figerait le paysage dans une immobilité minérale " (Gusdorf 1960 : 94). Ceci permet de mieux comprendre la fonction spécifique du rhétôr dans l'Antiquité, à savoir: défendre sa position publiquement, prendre parti par rapport à, contre, avancer des propositions, qualifier ou requalifier des faits, et d'abord s'engager à le faire, c'est-à-dire en assumer les risques. Et quels risques! Bien sûr, celui qui consiste à supporter la charge de la preuve - premier courage et premier devoir du citoyen en démocratie -, mais surtout celui qui naît de la suspension des jugements :

Critiquer [écrit Emmanuelle Danblon] revient à suspendre les jugements, une activité qui n'est pas spontanée et qui, plus que tout autre, doit faire l'objet d'un apprentissage, parce qu'elle est essentielle à la vie politique. Elle seule permet de garantir à l'homme sa liberté. Elle seule permet de laisser le monde ouvert. [...] La suspension des jugements permet à chacun de s'exercer à lutter contre son dogmatisme, contre sa peur de la liberté : contre sa propension, humaine, à la pesanteur. La critique est donc littéralement artificielle. Elle habitue le citoyen à placer ses opinions et ses jugements à bonne distance de sa personne afin de pouvoir les considérer plus objectivement : de se les figurer. (Danblon $2013: 21$ )

En s'engageant dans l'espace argumentatif, les adversaires savent qu'ils ont quelque chose à perdre dans l'exploration de leur différend; c'est d'ailleurs cette perte potentielle qui donne sens, corps et épaisseur à leur recherche concurrente de la persuasion. C'est parce qu'ils prennent ce risque que les contradicteurs font l'expérience de la liberté discursive autant que politique : celle de dire, celle d'adhérer ou de ne pas adhérer, c'est-à-dire, avant tout, cette liberté d'«inventer»- pour reprendre les termes de Perelman :

Nous pouvons considérer comme des manifestations de la liberté, d'une part, le comportement de celui qui invente soit des arguments à l'appui de sa thèse soit des objections contre la thèse adverse et, d'autre part, le comportement de celui qui se contente d'accorder ou de refuser son adhésion aux thèses qu'on lui présente. À la liberté d'invention, fondement de l'originalité, ferait pendant la liberté d'adhésion, fondement d'une communauté des esprits. (Perelman 1989 [1949] : 295)

L'entreprise rhétorique, tout entière, est inscrite dans la dualité de l'attaque et de la défense. Elle prend corps dans cette démarche constructive qui fait de la critique réciproque, critique responsable, une voie du progrès. Pour Danblon (2013: 145), la critique c'est précisément « l'affinage, [...] la sculpture des notions, des principes et des normes qu'une société se donne. » Or, dans une société ouverte, « une idée n'est jamais mieux éclairée que par son ombre portée», par son autre, son contraire. En conséquence de quoi : refuser à l'autre la possibilité même de la critique (comme si elle était forcément sans objet); se soustraire à la défense, et donc à la justification autrement qu'en rappelant l'évidence (du complot, en l'occurrence), cela revient à concevoir et à dénoncer l'accusation du contradicteur comme injustifiable ; à l'exclure du champ de l'argumentation.

Disons-le, la logique conspirationniste opère un renversement de la critique contre elle-même. Elle la détourne de sa fonction initiale et dévoie son esprit. D'un côté, elle prétend faire de l'entreprise critique (via la dénonciation publique d'une réalité cachée) son principe directeur, d'un autre côté, elle refuse la précarité à laquelle, justement, ouvre cette démarche. Or, il ne saurait y avoir d'argumentation bien entendue (orientée vers la critique comme vers la justification) sans reconnaissance de la précarité de l'objet sur lequel elle se porte ; reconnaissance de son caractère mouvant, 
relatif, non-absolu. L'argumentation n'a de sens - et sans doute de valeur aussi - que dans un monde où les arguments peuvent jouer parce qu'ils ne sont pas ligotés à la thèse, mais liés à un contexte, un public, des enjeux et une certaine hiérarchie des valeurs. Un monde où il y a du jeu, du souple, de l'incertain. Pour le dire autrement, toute « prise de position est précaire [parce que] toute argumentation s'insère dans un contexte [social et argumentatif] perpétuellement modifiable » (Perelman 1989 [1958]: 441). À cet égard, le théoricien du complot se comporte mutatis mutandis comme le philosophe absolutiste décrit (et dénoncé) par Chaïm Perelman : c'est parce qu'il veut à tout prix « échapper à la précarité d'une critique ou d'une justification relatives à des normes et à des fins particulières " qu'il «se met à la recherche de normes et de fins absolues, c'est-à-dire indiscutables " (Perelman 1990 [1966]: 413). On comprend pourquoi le principe de précarité (qui se trouve pourtant au cœur du travail argumentatif) va à l'encontre de la démarche conspirationniste qui cherche une sécurité cognitive absolue et une évacuation du doute.

Voyons maintenant (dans le prolongement de notre article: Danblon \& Nicolas 2012) quelques-uns des topoï propres aux discours conspirationnistes. Le but est ici de mieux saisir la clôture du sens et le refus de l'incertitude, du flou auxquels ces discours invitent, c'est-à-dire contraignent.

\section{Bref inventaire des topiques de la conspiration}

(1) «À qui profite le crime ? » C'est, me semble-t-il, le topos principal : celui qui, lancé à la cantonade mais avec un air entendu, permet de justifier l'enquête aux yeux mêmes des dénonciateurs du complot (dès lors convertis en nouveaux Sherlock Holmes), et donc de motiver la recherche, voire la traque tous azimuts des indices, des traces, des preuves. En disant principal, je ne veux pas dire que ce topos est premier. Ce qui est premier, c'est finalement de dire - une fois pour toutes - qu'un " crime » a été commis, ou mieux, de qualifier un événement quelconque comme tel. En cela, le topos en question, issu du roman noir ou d'espionnage, ne fonctionne qu'en association avec un autre lieu du discours qui légitime l'identification du crime comme de la recherche des criminels. Dans l'exemple suivant, l'auteur de l'accusation, tout en se défendant de formuler une accusation concrète (puisque " cela ne signifie rien »), se présente comme le bon citoyen qui invite à s'interroger, à se poser certaines questions, à faire marcher son esprit critique. Il ne condamne personne dit-il (même s'il donne des noms), il pose une question :

"Du nouveau pourrait sortir", assure Maurice Demolin, conseiller provincial et chef du groupe PS à Grâce-Hollogne. Comme secrétaire de la fédération liégeoise, il était en 1991 l'un des bras droits d'André Cools. Dans un livre récent, il évoque des "réunions secrètes", auxquelles auraient assisté des pontes du PS liégeois, juste avant et juste après l'assassinat. «Je n'aime pas la théorie du complot, explique-t-il. Je dis simplement ceci : cherchez à qui le crime profite. Du point de vue politique, il a notamment profité à Guy Mathot, à Guy Spitaels, à Jean-Maurice Dehousse et à José Happart. Attention, cela ne signifie rien. En disant ça, je n'accuse pas $»^{4}$.

Ici encore, à propos de l'affaire Mohammed Merah, le dénonciateur du complot - judéomaçonnique, en l'occurrence - pose cette question du crime (et de ses instigateurs) en la présentant sous les traits de la parfaite objectivité. La démarche de mise en « lumière », initiée par la question même, semble absolument désintéressée. Il s'agit (ou plutôt, il s'agirait), tout simplement, de « regarder» les faits, les crimes donc, sans $a$ 
priori, sans arrières pensées, sans œillères. L'entreprise de collecte des indices (lesquels, d'emblée, donnent la preuve que les coupables officiels ne sont pas les bons) parait guidée par un seul dessein : la recherche de la vérité. Or, celle-ci, forcément, n'est pas bonne à dire :

À qui profite le crime ? Si l'on regarde ces drames à la lumière froide de l'objectivité l'on peut faire resurgir des détails forts intéressants. Tous ces crimes deviennent politiques puisque les victimes sont issues de l'immigration, et que parmi elles, quatre sont juives, dont 3 enfants, exécutés dans une école juive. Armée, immigration, juifs... Trois composants modernes indissociables du mobile politique. Le tireur fou isolé n'a donc pas sa place ici, parmi les suspects à retenir. Par conséquent, le mobile politique est donc à privilégier plus que les autres, toujours sur la base de cette lumière froide de l'objectivité. [...] Objectivement, son crime instrumentalisé - ne peut que donner des voix en masse à nos deux candidats sionistes pour la présidence, à savoir : Sarkosy [sic] et Marine Le Pen; tous deux de farouches opposants à l'Islam, au nom de la laïcité maçonnique 5 .

(2) «Rien n'arrive par accident. » Le bon sens conspirationniste veut que tout ce qui arrive soit le fruit d'intentions et de projets humains (plans, protocoles, manigances, etc.). Ceci veut dire qu'il existe forcément des individus qui sont la cause des drames que subissent le monde et les hommes. Dans cette optique, accepter l'existence du hasard reviendrait à renoncer à son esprit critique. Plus précisément, ce serait renoncer à regarder par-delà ce que le hasard nous cache : à savoir la réalité. Dès lors, si le hasard s'apparente à une croyance, et même à une duperie, le simple fait de refuser le hasard, le flou et le précaire du monde devient un signe de clairvoyance et de lucidité. Le doute peut donc jouer partout, face à n'importe quoi ou n'importe qui, jusqu'à contaminer l'espace du discours tout entier. "Tout ce que je peux dire c'est que rien n'est fait au hasard, tout est fait de plans et de conspirations. Rien d'autres [sic $]^{6}{ }^{\prime}$.

(3) «Rien n'est tel qu'il parait être. » Tout est faux ou, plus exactement, « la vérité est ailleurs»- pour reprendre le slogan d'une série bien connue. Ce topos signale l'existence d'un feuilleté d'explications: depuis les «illusions» et les «contes pour enfants » propres aux explications officielles, jusqu'à la solide vérité des faits. Laquelle est forcément cachée au grand nombre. Le sens profond des choses n'est pas donné à tous; il n'est réservé qu'aux initiés, ceux qui, justement, savent que «tout n'est qu'imaginaire ».

Pourquoi risquer sa vie inutilement, et pour y faire quoi? En allant sur la lune? Alors que des images de cinéma d'intox à deux balles suffisent à convaincre. De Gagarine aux Américains sur la lune, tout n'est qu'imaginaire. Tout est faux, comme le reste ${ }^{7}$.

(4) «Tout est lié, des forces occultes tirent les ficelles. » Ce topos signale le caractère gigantesque du maillage et des ramifications du complot. Il se fonde sur une démarche indiciaire qui évolue en vase clos : en bas les ténèbres, en haut la lumière. En d'autres termes, il permet de faire le lien entre le caractère simple du grand récit suivant lequel « tout et lié » au sein du « jeu mondial » (" pas de chefs [...] que des exécutants »), et le côté complexe (voire même, le plus souvent, inquiétant) du système que ce grand récit met en scène sous la forme d'un "échiquier " ou d'une "toile d'araignée », par exemple. L'extrait suivant, tiré du film antimaçonnique Forces occultes, me paraît rendre bien compte de ce phénomène :

En Maçonnerie on cache tout aux petites gens. En bas vous ne savez rien... en haut vous commencez à voir un peu plus clair dans le jeu mondial. [...] Y'a pas de chefs, 
chez nous. Il n'y a que des exécutants. Je ne suis qu'un pion sur un échiquier...

j'accomplis une fonction... je reçois des messages... j'obéis... je transmets... j'agis ${ }^{8} . .$.

31 (5) «Qu'on me prouve le contraire!» Ainsi se manifeste le renversement de la charge de la preuve. Il n'appartient plus à celui qui accuse de prouver ce qu'il avance (d'en donner les raisons ; de se justifier), mais à ceux qui sont accusés d'apporter la preuve, et plus encore la preuve irréfragable (laquelle est en fait impossible à fournir), qu'ils ne complotent pas. Ce topos témoigne justement d'une renonciation aux risques propres à l'entreprise rhétorique ; propres à la critique et à la justification. Dans l'exemple choisi, on remarquera d'ailleurs la combinaison de deux postures éthiques (ethos) : celle de l'homme sans qualités ("je ne suis pas bardé de diplômes, j'ai 61 ans »), et celle de l'homme qui ne fait que s'interroger à bon droit et qui, finalement, n'est pas plus bête que les médias ni moins sensé que les puissants («vous ne trouvez pas bizarre [...] comme c'est bizarre [...] je sais encore réfléchir ») :

Vous ne trouvez pas bizarre la façon dont ça [a] été expédié par contre pour détruire DSK ils ont mis le paquet c'est lamentable, on se croirait dans un vilain polar, depuis 15 jours déjà les médiats [sic] nous parlaient DSK par ci DSK par là comme c'est bizarre, en tout qu'à [sic] il fallait l'achever et là c'est réussit [sic] honte aux protagonistes d'avoir monté toute cette affaire, je ne suis pas bardé de diplômes, j'ai 61 ans mais je sais encore réfléchir aussi je le dis tout ça c'est un complot qu'on me prouve le contraire ${ }^{9}$ !

\section{Conclusion}

Pour refermer cette réflexion, je soulignerai d'abord que la pensée conspirationniste constitue un remarquable laboratoire pour aborder, par contraste, la pratique rhétorique, c'est-à-dire pour mieux comprendre sa valeur profonde, ses usages autant que ses outils. Pour le dire d'un trait, l'argumentation mobilisée pour soutenir ce type d'explications totalisantes est un "faux-semblant», parce que, dans tous les cas, les « jeux sont faits ». Il n'y a rien à défendre ni à risquer. Le monde est clos; les preuves sont irréfutables; le doute n'a plus lieu d'être ; la vérité est là - sous nos yeux. Dès lors, la seule voie disponible et raisonnable demeure l'adhésion inconditionnelle aux thèses avancées. Tout le reste n'est que mauvais esprit, aveuglement ou complicité.

33 En même temps, s'il convient de "faire semblant" d'argumenter (d'apporter des raisons et de donner des preuves), c'est que l'argumentation, en tant que telle, a de la valeur et constitue un moyen de se légitimer et de renforcer l'édifice explicatif. Comme le souligne Perelman, « [c']est parce qu'une monnaie est en circulation et possède une valeur que l'on se donne la peine de faire semblant de fabriquer de la fausse monnaie » (Perelman 1989 [1959] : 371). Au reste, l'analyse du discours conspirationniste, tout spécialement dans ses rapports au doute et à la critique, informe notre conception de la liberté et sert à préciser les conditions de son exercice responsable.

Toutefois, pour saisir leur succès, leur attractivité et leur efficacité, il faut se garder d'aborder ces théories-là (aussi exotiques soient-elles) comme le témoignage d'une pensée folle ou inculte - en d'autres termes comme irrationnelles. Elles signalent bien plutôt la résurgence non assumée et souvent désavouée de raisonnements archaïques (par indices, par intuition, etc.) à l'intérieur de la modernité (Danblon 2010). En somme, "elles interpellent en chacun de nous [...] une forme de rationalité cognitive» (Bronner 2009: 66-67) qui n'est pas insensée, mais qui manifeste aussi un manque 
d'exercice, un dévoiement et sans doute un retournement contre elle-même de l'activité argumentative.

\section{BIBLIOGRAPHY}

Aristote. 1990. Éthique à Nicomaque, trad. Jules Tricot (Paris : Vrin)

Aristote. 1960. Rhétorique, t. I, trad. Médéric Dufour (Paris : Les Belles Lettres)

Bronner, Gérald. 2009. La pensée extrême. Comment des hommes ordinaires deviennent des fanatiques

(Paris : Denoël Impacts)

Danblon, Emmanuelle. 2013. L'homme rhétorique. Culture, raison, action (Paris : Cerf)

Danblon, Emmanuelle. 2010. « Les "théories du complot" ou la mauvaise conscience de la pensée moderne », Danblon, Emmanuelle, Loïc Nicolas (éds). Les rhétoriques de la conspiration (Paris : CNRS Éditions), 57-72

Danblon, Emmanuelle \& Loïc Nicolas. 2012. « Rhétorique et topique de la conspiration », Raison publique $16,33-42$

Danblon, Emmanuelle \& Loïc Nicolas. 2010. Les rhétoriques de la conspiration (Paris : CNRS Éditions)

Dominicy, Marc. 2010. «Les sources cognitives de la théorie du complot. La causalité et les faits », Danblon, Emmanuelle \& Loïc Nicolas (éds). Les rhétoriques de la conspiration (Paris : CNRS Éditions), 119-132

Gusdorf, Georges. 1960. La vertu de force (Paris : PUF)

McEvoy, Sebastian. 1995. L'invention défensive. Poétique, linguistique, droit (Paris : Éditions Métailié) Nicolas, Loïc. 2010. « Rhétorique du complot : la persuasion à l'épreuve d'elle-même. Itinéraire d'une pensée fermée », Danblon, Emmanuelle \& Loïc Nicolas (éds). Les rhétoriques de la conspiration (Paris : CNRS Éditions), 73-96

Perelman, Chaïm. 2009 [1949]. « Le libre examen, hier et aujourd'hui », Modernité du libre examen. Textes de Chaïm Perelman et Jean Stengers, Préface de Jean-Pierre Devroey (Bruxelles : Éditions de l'Université de Bruxelles), 137-147

Perelman Chaïm. 1990 [1966]. « Considérations sur la raison pratique », Éthique et droit (Bruxelles : Éditions de l'Université de Bruxelles), p. 406-419

Perelman Chaïm, 1989 [1949]. « Liberté et raisonnement », Rhétoriques (Bruxelles : Éditions de l'Université de Bruxelles), 295-299

Perelman Chaïm. 1989 [1958]. « De la temporalité comme caractère de l'argumentation », Rhétoriques (Bruxelles : Éditions de l'Université de Bruxelles), 437-467

Perelman Chaïm. 1989 [1959]. « Les cadres sociaux de l'argumentation », Rhétoriques (Bruxelles : Éditions de l'Université de Bruxelles), 359-381

Perelman, Chaïm. 1952 [1948]. « Le problème du bon choix », Rhétorique et philosophie. Pour une théorie de l'argumentation en philosophie (Paris, PUF), 142-160 
Perelman, Chaïm \& Lucie Olbrechts-Tyteca. 1952 [1950]. « Logique et rhétorique », Rhétorique et philosophie. Pour une théorie de l'argumentation en philosophie (Paris : PUF), 1-43

Perelman, Chaïm \& Lucie Olbrechts-Tyteca. 1988 [1958]. Traité de l'Argumentation. La Nouvelle rhétorique (Bruxelles : Éditions de l'Université de Bruxelles)

\section{NOTES}

1. La suite du billet vient préciser les termes du prétendu complot entre Hitler, les sionistes et les tenants de ladite "Révolution mondiale" (sans doute les francs-maçons) en vue de créer l'État d'Israël : « Hitler's smear campaign was exclusively against Jews, but he had concealed the names of the Zionists who, with greatest brutality and disregard for the Jewish community, had planned the World Revolution and the foundation of the State of Israel. This is striking evidence that he worked together with the Zionists and that he was a first class liar, hypocrite and public traitor. » http://www.henrymakow.com/adolf_hitler_--_agent_of_zioni.html (consulté le 6 janvier 2014).

2. En-tête de l'article « Complots mondial [sic], délires, vraiment ? » publié le 2 janvier 2014 sur le Blog «Blida Éveil»: http://blideodz.wordpress.com/2014/01/02/complots-mondial-deliresvraiment/ (consulté le 6 janvier 2014).

3. Tribune libre mise en ligne par Mathieu Kassovitz le 11 septembre 2011. Vidéo consultée le 6 janvier 2014 sur le site : http://www.agoravox.tv/tribune-libre/article/mathieu-kassovitz-parledu-11-31679.

4. Propos tenus par Maurice Demolin (un politicien belge) et rapportés dans un article du journal Le Vif: "André Cools: la théorie du complot politique garde des partisans", par François Brabant, 18 juillet 2011.

5. Extrait de l'« Essai sur une théorie du complot: "De Naplouse à Toulouse" », par Emmanuel Lecomte, consulté le 3 janvier 2014 sur le site Iran French Info: http://french.irib.ir/info/ sant\%C3\%A9/item/177408-essai-sur-une-th\%C3\%A9orie-du-complot---de-naplouse-\%C3\%A0toulouse--par-emmanuel-lecomte

6. Message posté par «lyly» le 17 janvier 2009 sur le blog «Area51»: http:// area51blog.wordpress.com/2008/-03/28/les-attentats-du-11-septembre-a-qui-profite-le-crime/ (consulté le 6 janvier 2014).

7. Message posté le 29 décembre 2008 sur le site: http://conquistador-space.over-blog.fr/ (consulté le 6 janvier 2014).

8. Extrait du film Forces occultes de Paul Riche (pseudonyme de Jean Mamy), Nova films, 1943.

9. Message posté le 19 mai 2011 par «moune » sur un blog du journal Le Monde (consulté le 6 janvier 2014): http://bigbrowser.blog.lemonde.fr/2011/05/19/complot-dsk-se-serait-plaintdune-alliance-franco-russe-pour-le-virer-du-fmi/

\section{ABSTRACTS}

This paper deals with the place, status and ambiguities of doubt in conspiracy theories. Its aim is to question, with Chaim Perelman, the argumentative properties and topical basis of these theories through the three terms: evidence, doubt, and critique. In conspiratorial perspectives, 
doubt invades the social sphere: the world is only an appearance, lies are everywhere. However, inside the discourse itself, no doubt is possible: evidence is the law. So, the critical approach that structures and nourishes the conspiracy theories, namely the act of denunciation, is diverted from its original purpose and spirit.

Le but de cet article est de questionner, avec Chaïm Perelman notamment, la place et le statut du doute dans la pensée conspirationniste afin d'en souligner les ambiguïtés. Il s'agit plus encore de reconsidérer, à la lumière du triptyque doute-évidence-critique, le type d'argumentation et le fond topique qui caractérisent cette pensée-là. Alors que le doute envahit tout l'espace social car le monde n'est qu'apparences trompeuses; le mensonge est partout -, il en vient à quitter totalement celui du discours à l'intérieur duquel, justement, on ne doute pas. En effet, l'évidence y fait loi. Partant, l'entreprise critique, qui structure et nourrit la dénonciation du complot, se trouve détournée de sa fonction initiale et de son esprit.

INDEX

Mots-clés: doute, évidence, monde clos, Perelman, rhétorique, théories du complot, topoï

Keywords: closed world, conspiracy theories, doubt, evidence, Perelman, rhetoric, topoï

\section{AUTHOR}

\section{LOÏC NICOLAS}

Université Libre de Bruxelles, GRAL 